\title{
Molecular identification of Echinococcus granulosus on the Tibetan Plateau using mitochondrial DNA markers
}

D. Hu ${ }^{1}$, X. Song ${ }^{1}$, N. Wang ${ }^{1}$, X. Zhong ${ }^{1}$, J. Wang ${ }^{1}$, T. Liu', Z. Jiang ${ }^{2}$, T. Dawa ${ }^{3}$, X. Gu${ }^{1}$, X. Peng ${ }^{4}$ and G. Yang ${ }^{1}$

1'Department of Parasitology, College of Veterinary Medicine, Sichuan Agricultural University, Ya'an, China

${ }^{2}$ Ganzi Animal Science Institute, Kangding, China

${ }^{3}$ Tibetan Center for Animal Disease Control and Preventive, Lasa, China

${ }^{4}$ Department of Chemistry, College of Life and Basic Science,

Sichuan Agricultural University, Ya'an, China

Corresponding author: G. Yang

E-mail: guangyou1963@aliyun.com

Genet. Mol. Res. 14 (4): 13915-13923 (2015)

Received January 7, 2015

Accepted July 29, 2015

Published October 29, 2015

DOI http://dx.doi.org/10.4238/2015.October.29.12

ABSTRACT. Cystic echinococcosis (CE) is an important worldwide zoonotic disease that causes large economic losses and human suffering. Echinococcus granulosus, the causative agent of $\mathrm{CE}$, exhibits different genotypes in different locations. In order to identify its genotypes and analyze its genetic structure on the Tibetan Plateau, we collected 72 hydatid cysts from different intermediate hosts and amplified and sequenced their mitochondrial cytochrome c oxidase subunit 2 (cox2) genes. Seventy isolates were identified as the $E$. granulosus G1 genotype, while two isolates belonged to the $\mathrm{G} 6$ genotype. There were 18 haplotypes among the $70 \mathrm{E}$. granulosus isolates, which exhibited a star-like network pattern and shared a common haplotype $\left(H_{1}\right)$. There was little difference between geographical sub-populations. Our results suggest that a recent $E$. granulosus population expansion occurred 
on the Tibetan Plateau, suggesting that E. granulosus was introduced into China. This study increases the basic molecular data needed for the molecular diagnosis, epidemiology, prevention, and control of Echinococcus diseases.

Key words: Echinococcus granulosus; Mitochondrial DNA; Tibetan Plateau; Molecular identification

\section{INTRODUCTION}

Cystic echinococcosis (CE) is a global zoonotic disease caused by the infection of mammals by the larval (metacestode) stage of Echinococcus granulosus. It causes 1 million disability-adjusted life years in humans and an annual loss of 2 billion US dollars in livestock (Budke et al., 2006). Recently, the World Health Organization has included CE as part of a neglected zoonosis subgroup for its strategic plans for the control of neglected tropical diseases (Siracusano et al., 2011).

The metacestode stage of $E$. granulosus inhabits the liver, lungs, and other internal organs of livestock and humans after oral uptake of the eggs, which are produced by adult worms in the canine small intestine (Moro and Schantz, 2009; McManus et al., 2012). Previous studies have identified 10 genotypes of E. granulosus (G1 to G10) (Bowles et al., 1992; Bowles et al., 1994; Scott et al., 1997; Lavikainen et al., 2003), but it is currently considered as a complex that consists of at least four species: E. granulosus sensu strictu (genotypes G1 to G3), E. equinus (G4), E. ortleppi (G5), and E. canadensis (G6 to G10); however, the species status of E. canadensis is ambiguous (Nakao et al., 2010b; McManus, 2013; Nakao et al., 2013).

Mitochondrial DNA has many specific characteristics, such as matrilineal inheritance and no intron structure, which make it an important tool in population evolution and taxonomy studies. Given that different DNA markers have different rates of evolution and conserved sites, the results obtained from analyzing the same samples may be inconsistent. The mitochondrial cytochrome $\mathrm{c}$ oxidase subunit 2 (cox2) gene has been widely used in studies of evolution and genetic diversity in many species (Frati et al., 1997; Rawson and Burton, 2006), but not in E. granulosus. In this study, we investigated whether cox 2 is a suitable marker by sequencing its complete fragments and analyzing the genetic structure and phylogenetic relationships of $E$. granulosus on the Tibetan Plateau. This information will provide basic molecular data for the molecular diagnosis, epidemiology, prevention, and control of CE in this region.

\section{MATERIAL AND METHODS}

\section{Samples}

A total of 72 hydatid cyst isolates were collected in 2011 and 2013 from humans, sheep, and yaks on the Tibetan Plateau in the Sichuan and Qinghai provinces of southeastern China and in the Tibet Autonomous Region (Table 1). All of the animal isolates were collected from slaughterhouses, while human samples were obtained from the People's Hospital of Ganzi County in Sichuan province. Cysts were washed three times in normal saline and subsequently stored at $-80^{\circ} \mathrm{C}$ until use. 


\begin{tabular}{|c|c|c|c|}
\hline & Sichuan & Tibet & Qinghai \\
\hline Sheep & - & 12 & 35 \\
\hline Human & 23 & - & - \\
\hline Yak & 1 & 1 & - \\
\hline Total & 24 & 13 & 35 \\
\hline
\end{tabular}

\section{DNA extraction and polymerase chain reaction (PCR)}

Genomic DNA from each isolate was extracted by the phenol-chloroform method (Jacobs et al., 1997). A complete cox2 gene fragment was amplified from each sample by $P C R$ using forward (Ps, 5'-TGAGGTAAGTCGTAACAAGG-3') and reverse (Pa, 5'-ATCTACAGCACGAAAAGCC-3') primers. Both primers were designed based on a standard sheep strain (GenBank accession No. AF297617) using Primer Premier software version 5.0 (Premier Biosoft International, CA, USA). The PCR was conducted in a final volume of $20 \mu \mathrm{L}$, which contained $10 \mu \mathrm{L} 2 \mathrm{X}$ Taq PCR Master Mixture (Tiangen, Beijing, China), $1 \mu \mathrm{L}$ genomic DNA, $1 \mu \mathrm{L}$ of each primer, and $8 \mu \mathrm{L} \mathrm{ddH}_{2} \mathrm{O}$. The PCR program consisted of one cycle of primary denaturation $\left(5 \mathrm{~min}\right.$ at $95^{\circ} \mathrm{C}$ ) followed by 39 cycles of denaturation $\left(30 \mathrm{~s}\right.$ at $\left.94^{\circ} \mathrm{C}\right)$, annealing $\left(45 \mathrm{~s}\right.$ at $\left.50^{\circ} \mathrm{C}\right)$, extension $\left(45 \mathrm{~s}\right.$ at $\left.72^{\circ} \mathrm{C}\right)$, and a final extension $\left(10 \mathrm{~min}\right.$ at $72^{\circ} \mathrm{C}$ ). Positive and negative (no DNA) controls were included with each PCR set. To assess the quality of the PCR amplicons, $8 \mu \mathrm{L}$ of each PCR product were run on $1.0 \%(\mathrm{w} / \mathrm{v})$ agarose gels. The PCR products were purified using a TIANgel Midi Purification Kit (Tiangen), and were then sequenced in two directions by the Invitrogen Trading (Shanghai) Co. Ltd. Reference sequences were retrieved from the National Center for Biotechnology Information (http://www.ncbi.nlm.nih.gov).

\section{Data analysis}

Nucleotide sequences were aligned and compared using DNAMAN software (Version 5.2.2.0, Lynnon Biosoft, Quebec, Canada). Amino acid sequences were inferred from the nucleotide sequences using the flatworm mitochondrial genetic code (Nakao et al., 2000) in MEGA version 5.05 (Tamura et al., 2011). Population diversity indices, including the number of haplotypes $\left(H_{N}\right)$, haplotype diversity $\left(H_{D}\right)$, and nucleotide diversity $(\pi)$, were calculated using DnaSP 5.10 (Librado and Rozas 2009). Maximum likelihood (ML) and Bayesian trees were constructed using MEGA and MrBayes version 3.1.2 (Ronquist and Huelsenbeck, 2003), respectively, on the default settings. TCS 1.2 software (Clement et al., 2000) was used to build an E. granulosus haplotype network by statistical parsimony. The neutrality indices Tajima's $D$ (Tajima 1989) and Fu's $F_{S}$ (Fu 1997) were calculated using the population genetics package Arlequin version 3.5.1.2 (Excoffier and Lischer 2010).

For the geographical sub-population analysis, the nucleotide sequences were grouped into three populations: Qinghai, Sichuan, and Tibet. Population diversity indices, including the number of segregating sites $(S), H_{N}, H_{D}$, and $\pi$, were estimated using DnaSP 5.10. Tajima's $D$ and Fu's $F_{S}$ were also calculated for each sub-population. Pairwise genetic differences between the three sub-populations were estimated using Wright's F-statistic $\left(F_{S T}\right)$ by an analysis of molecular variance (AMOVA) in Arlequin, and the degree of gene flow $\left(N_{M}\right)$ was also calculated. The average number of pairwise nucleotide differences $\left(K_{X Y}\right)$, the nucleotide substitution per site $\left(D_{X Y}\right)$, and the net nucleotide substitution per site $\left(D_{A}\right)$ between the sub-populations were calculated using DnaSP. 


\section{Ethics}

Human hydatid cysts were kindly donated by the hospital's Center for Genetic Variation Studies and all of the samples were anonymous. According to hospital policy, patients were required to sign informed consent forms for the surgical procedure. The research procedures followed were in accordance with the Helsinki Declaration of 1975, as revised in 2008.

Animals were handled in accordance with the Animal Protection Law of the People's Republic of China (a draft of the Animal Protection Law was released in China on September 18, 2009). This study was approved by the National Institute of Animal Health, Animal Care, and Use Committee of Sichuan Agricultural University (Approval No. 2011-015).

\section{RESULTS}

\section{Nucleotide variability and polymorphisms}

All 72 isolates were successfully amplified for the complete cox 2 mitochondrial gene; of these, two isolates from Tibetan sheep (length $576 \mathrm{bp}$ ) shared the same sequence (GenBank accession Nos. KC692991 and KC692992) and exhibited a high identity (99.83\%) to a previously characterized camel strain (GenBank accession No. AB208063). We identified these two isolates as E. canadensis (genotype $\mathrm{G} 6$ and haplotype $19\left[\mathrm{H}_{19}\right]$ ).

The remaining 70 isolates (GenBank accession Nos. KC692921 to KC692990, length 582 bp, and encoding 193 amino acids) exhibited a high identity (99.48 to 99.83\%) to a standard sheep strain (GenBank accession No. AF297617), and were identified as E. granulosus sensu strictu (G1 genotype, see Table S1). Multiple sequence alignments revealed 18 haplotypes $\left(H_{1}-H_{18}\right)$ and 17 variation sites, in which 10 were single mutation sites and seven were parsimony informative sites. Five non-synonymous mutations (29.4\%) were also found in the amino acid sequences (Table S2). Only nucleotide substitutions were detected; deletions and insertion mutations were not observed.

\section{Phylogenetic analysis and network}

The ML and Bayesian trees of these haplotypes shared the same topological structure. According to these phylogenetic relationships, all of the sequences could be classified into two main branches: the G1 genotype and other genotypes of $E$. granulosus. Haplotypes $\mathrm{H}_{1}$ to $\mathrm{H}_{18}$ were identified as having the $\mathrm{G} 1$ genotype, while $\mathrm{H}_{19}$ belonged to the $\mathrm{G} 6$ genotype (Figure 1). The haplotype network exhibited a star-like expansion, with a common haplotype $\left(H_{1}\right)$ at its center (Figure 2); only one or two mutation steps were detected between the common haplotype and the others. Ten, four, and three geographically unique haplotypes were found in Qinghai, Sichuan, and Tibet, respectively.

\section{Sub-population genetic diversity indices}

Population diversity indices were calculated using the nucleotide sequences of the cox2 gene from the Tibetan Plateau (Table 2). The $H_{D}$ and $\pi$ of the sequences were $0.667 \pm 0.064$ and $0.00148 \pm 0.00020$, respectively. Among the sub-populations, the highest $H_{D}$ and $\pi$ values were obtained in Qinghai. Overall, Tajima's $D$ and Fu's $F_{s}$ were both significant and negative, but 
among the sub-populations only the Tajima's $D$ value for Qinghai was significant and negative. The AMOVA revealed that $97.21246 \%$ of the variation was within the sub-populations and only $2.78754 \%$ was between them.

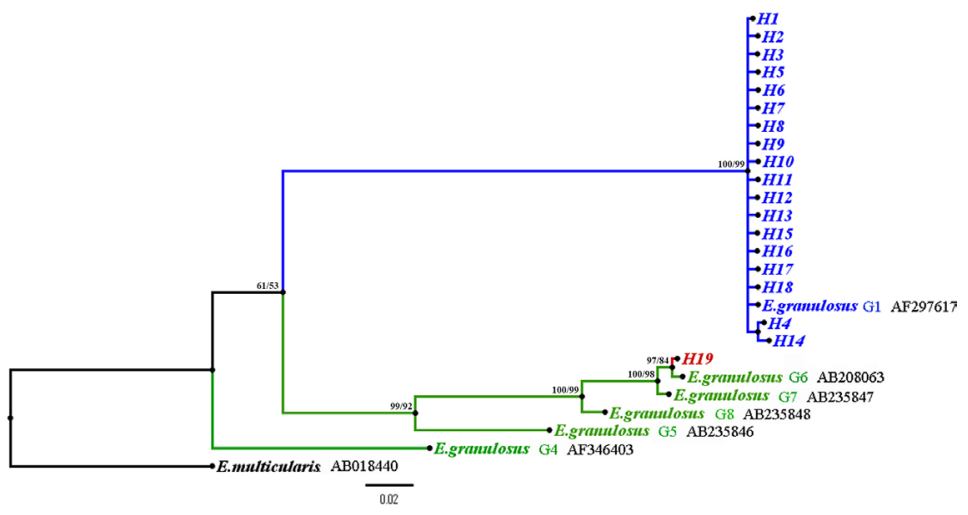

Figure 1. Phylogenetic tree of Echinococcus granulosus haplotypes based on maximum likelihood and Bayesian methods. GenBank accession numbers for reference sequences are stated after the species name. Bootstrap values of Bayesian (first value) and maximum likelihood (second value) are stated above each branch.

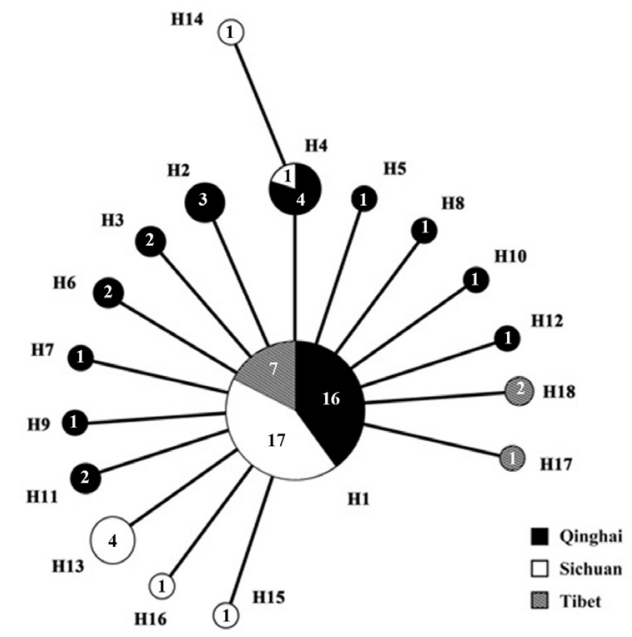

Figure 2. Echinococcus granulosus haplotype network on the Tibetan Plateau. Values in circles represent the haplotype distribution frequency.

Table 2. Diversity and neutrality indices of Echinococcus granulosus sub-populations.

\begin{tabular}{lcrrcccc}
\hline & No. & $S$ & $H_{N}$ & $H_{D} \pm \mathrm{SD}$ & $\pi \pm \mathrm{SD}$ & Tajima's $D$ & Fu's $F_{S}$ \\
\hline Qinghai & 35 & 11 & 12 & $0.778 \pm 0.068$ & $0.00180 \pm 0.00025$ & $-1.896^{*}$ & -2.070 \\
Sichuan & 24 & 5 & 5 & $0.486 \pm 0.113$ & $0.00107 \pm 0.00033$ & -1.549 & -2.309 \\
Tibet & 11 & 3 & 4 & $0.600 \pm 0.154$ & $0.00119 \pm 0.00038$ & -1.114 & -1.525 \\
Tibetan Plateau & 70 & 17 & 18 & $0.667 \pm 0.064$ & $0.00148 \pm 0.00020$ & $-2.24115^{\star \star *}$ & $-19.827^{* *}$ \\
\hline
\end{tabular}

${ }^{*} \mathrm{P}<0.05 ;{ }^{* *} \mathrm{P}<0.02 ;{ }^{* * *} \mathrm{P}<0.01$. No., number of sequences; $S$, number of segregating sites; $H_{N}$, number of haplotypes; $H_{D}$, haplotype diversity; $\pi$, nucleotide diversity. 
The $K_{X Y}$ values ranged between 0.67636 and 0.00116 (Sichuan and Tibet, respectively), and the $D_{X Y}$ values varied between 0.88571 and 0.00152 (Qinghai and Tibet, respectively) (Table 3). $F_{S T}$ values between the sub-populations ranged between 0.00646 (Tibet and Qinghai) and 0.05026 (Tibet and Sichuan), with $N_{M}$ values of 39.96235 (Tibet and Qinghai) and 4.72440 (Tibet and Sichuan) (Table 4). There was very low genetic differentiation ( $G_{\mathrm{ST}}, 0.01051$ to $0.03426 ; F_{\mathrm{ST}}$, 0.00646 to 0.05026$)$ and very high gene flow $\left(N_{M}, 4.72440\right.$ to 39.96235$)$ between the three subpopulations.

\begin{tabular}{|c|c|c|c|c|c|}
\hline Population 1 & Population 2 & $K_{X Y}$ & $D_{X Y}$ & $D_{A}$ & $G_{\text {ST }}$ \\
\hline Sichuan & Qinghai & 0.85371 & 0.00147 & 0.00005 & 0.03426 \\
\hline Sichuan & Tibet & 0.67636 & 0.00116 & 0.00005 & 0.01051 \\
\hline Qinghai & Tibet & 0.88571 & 0.00152 & 0.00003 & 0.01273 \\
\hline
\end{tabular}

$K_{X Y}$, average proportion of nucleotide differences between sub-populations; $D_{X Y}$, average number of nucleotide substitutions per site between sub-populations; $D_{A}$, number of net nucleotide substitutions per site between subpopulations; $G_{S T}$, genetic differentiation index based on haplotype frequency.

\begin{tabular}{|c|c|c|c|}
\hline & Qinghai & Sichuan & Tibet \\
\hline Qinghai & & 7.39760 & 39.96235 \\
\hline Sichuan & $0.03269^{* * *}$ & & 4.72440 \\
\hline Tibet & 0.00646 & 0.05026 & \\
\hline
\end{tabular}

${ }^{* * *} \mathrm{P}<0.01$

\section{DISCUSSION}

E. granulosus is one of the most important parasites in the world, because it affects human health and causes large economic losses. E. granulosus is spread over 27 provinces, autonomous regions, and municipalities in China, and its main areas of endemism are western and northwestern parts of the country. Recent studies that have conducted the molecular identification of $E$. granulosus found that the $\mathrm{G} 1$ genotype is the main genotype in this region, and there are a small number of the G3, G6, and G7 genotypes (Cardona and Carmena, 2013; Wang et al., 2014b; Zhang et al., 2014). In this study, 70 isolates were identified as the E. granulosus G1 genotype, while two isolates (both derived from sheep in Tibet) belonged to the G6 genotype. The two genotypes on the Tibetan Plateau in southeastern China are consistent with the epidemiological characteristics of human and animal CE in China.

A number of mitochondrial and nuclear fragments have been used as markers to investigate the genotypes, or the genetic structures, of $E$. granulosus in southwestern China, including cox1, nad1, nad2, cytb, atp6, 12S rRNA, and 16S rRNA (Ma et al., 2008; Nakao et al., 2010a; Ma et al., 2012; Wang et al., 2013; Yan et al., 2013; Wang et al., 2014a; Zhong et al., 2014). However, markers of different lengths are not appropriate for the comparison of genetic structures in different regions, and gene fragments may not be representative because every fragment on a gene is unique in terms of its conservation characteristics. Therefore, we amplified the cox 2 gene as a complete sequence, and identified 18 haplotypes of the E. granulosus $\mathrm{G} 1$ genotype from 70 
isolates and found that 40 isolates shared $100 \%$ homology with each other, demonstrating that cox2 is highly conserved. These results suggest that cox2 could be a candidate marker for the genetic study of $E$. granulosus.

The genetic structures of E. granulosus in the Middle East (Iran and Jordan), South America (Peru), and Southeast Asia (China) differ (Casulli et al., 2012; Yanagida et al., 2012). According to previous studies (Nakao et al., 2010a; Ma et al., 2012; Wang et al., 2013; Yan et al., 2013; Wang et al., 2014a), the characterization of The E. granulosus genetic network in Southeast Asia is characterized by a star-like pattern, with a predominance of the G1 genotype except in India, where the G3 genotype predominates (Sharma et al., 2013). We also found a radial network based on a common haplotype, but there were few mutation steps between the common haplotype and the others. This may due to the highly conserved nature of cox2 and the low diversification of $E$. granulosus on the Tibetan Plateau. In addition, E. granulosus is a diploid organism that has a mixed sexual and asexual reproduction system. Adult worms are hermaphroditic and exhibit sexual reproduction, while the larval stage is asexual (Haag et al., 1999); this may result in a low level of nucleotide locus variability.

Despite the fact that the environments in Qinghai, Sichuan, and Tibet differ considerably, the inter-population comparison estimates $\left(K_{X Y}, D_{X Y}, G_{S T}\right.$, and $\left.F_{S T}\right)$ and AMOVA revealed a low level of genetic differentiation. This may have been caused by their common haplotype and the high level of gene flow between these sub-populations, as well as their low level of diversity. All of our results suggest that the three sub-populations have not evolved into distinct populations.

Neutrality tests, such as Tajima's $D$ and Fu's $F_{s}$, have been developed to test the selective neutrality of nucleotide mutations, and are used to determine population growth. The significant, negative values of Tajima's $D$ and Fu's $F_{S}$ we obtained indicate an excess of rare alleles, which could be a signature of recent population expansion (Tajima, 1989; Fu, 1997). Sheep were first domesticated in the Middle East around 12,000 BC, before spreading into Europe, Africa, the Americas, and Asia. Recent studies have hypothesized that sheep parasites also spread, which caused a parasite population expansion in these regions (Casulli et al., 2012; Yanagida et al., 2012; Sharma et al., 2013). Nakao et al (2010) conjectured that E. granulosus spread into Peru from Europe due to livestock importation during the 15th century. We suggest that the "Silk Road" between China and the Middle East accelerated the spread of this parasite by the trading of domestic animals in the 1st century. This hypothesis is consistent with Nakao et al.'s (2010b) timeline; however, archaeoparasitological evidence, molecular epidemiological studies, and comparisons of $E$. granulosus genetic structures in different regions are required to confirm this hypothesis.

In this study, we confirmed that the E. granulosus G1 genotype is the predominant genotype in China by using complete cox 2 sequences of 72 samples from the Tibetan Plateau, and a low level of differentiation was found between three geographical sub-populations. In order to fully understand $E$. granulosus transmission, further comparative studies of its genetic structure in different regions should be conducted.

\section{Conflicts of interest}

The authors declare no conflict of interest.

\section{ACKNOWLEDGMENTS}

Research supported by grants from the National Key Technology Research and Development Program of China (\#2006BAI06B09) and the Science and Technology Projects of 
the General Administration of Quality Supervision, Inspection, and Quarantine of the People's Republic of China (\#2009IK019). The authors thank the People's Hospital of Ganzi County and Shijin Deng for providing the samples.

\section{Supplementary material}

\section{REFERENCES}

Bowles J, Blair D and McManus DP (1992). Genetic variants within the genus Echinococcus identified by mitochondrial DNA sequencing. Mol. Biochem. Parasitol. 54: 165-173.

Bowles J, Blair D and McManus DP (1994). Molecular genetic characterization of the cervid strain ('northern form') of Echinococcus granulosus. Parasitology 109: 215-221.

Budke CM, Deplazes P and Torgerson PR (2006). Global socioeconomic impact of cystic echinococcosis. Emerg. Infect. Dis. 12: 296-303.

Cardona GA and Carmena D (2013). A review of the global prevalence, molecular epidemiology and economics of cystic echinococcosis in production animals. Vet. Parasitol. 192: 10-32.

Casulli A, Interisano M, Sreter T, Chitimia L, et al. (2012). Genetic variability of Echinococcus granulosus sensu stricto in Europe inferred by mitochondrial DNA sequences. Infect. Genet. Evol. 12: 377-383.

Clement M, Posada D and Crandall KA (2000). TCS: a computer program to estimate gene genealogies. Mol. Ecol. 9: 1657-1659.

Excoffier $L$ and Lischer HE (2010). Arlequin suite version 3.5: a new series of programs to perform population genetics analyses under Linux and Windows. Mol. Ecol. Resour. 10: 564-567.

Frati F, Simon C, Sullivan J and Swofford DL (1997). Evolution of the mitochondrial cytochrome oxidase II gene in Collembola. J. Mol. Evol. 44: 145-158.

Fu Y (1997). Statistical tests of neutrality of mutations against population growth, hitchhiking and background selection. Genetics 147: 915-925.

Haag KL, Araujo AM, Gottstein B, Siles-Lucas M, et al. (1999). Breeding systems in Echinococcus granulosus (Cestoda: Taeniidae): selfing or outcrossing? Parasitology 118: 63-71.

Jacobs DE, Zhu X, Gasser RB and Chilton NB (1997). PCR-based methods for identification of potentially zoonotic ascaridoid parasites of the dog, fox and cat. Acta Trop. 68: 191-200.

Lavikainen A, Lehtinen M, Meri T, Hirvela-Koski V, et al. (2003). Molecular genetic characterization of the Fennoscandian cervid strain, a new genotypic group (G10) of Echinococcus granulosus. Parasitology 127: 207-215.

Librado P and Rozas J (2009). DnaSP v5: a software for comprehensive analysis of DNA polymorphism data. Bioinformatics 25: 1451-1452.

Ma S, Maillard S, Zhao H, Huang X, et al. (2008). Assessment of Echinococcus granulosus polymorphism in Qinghai province, People's Republic of China. Parasitol. Res. 102: 1201-1206.

Ma J, Wang H, Lin G, Craig PS, et al. (2012). Molecular identification of Echinococcus species from eastern and southern Qinghai, China, based on the mitochondrial cox1 gene. Parasitol. Res. 111: 179-184.

McManus DP (2013). Current status of the genetics and molecular taxonomy of Echinococcus species. Parasitology 140: $1617-1623$.

McManus DP, Gray DJ, Zhang W and Yang Y (2012). Diagnosis, treatment, and management of echinococcosis. BMJ 344 : e3866.

Moro P and Schantz PM (2009). Echinococcosis: a review. Int. J. Infect. Dis. 13: 125-133.

Nakao M, Sako Y, Yokoyama N, Fukunaga M, et al. (2000). Mitochondrial genetic code in cestodes. Mol. Biochem. Parasitol. 111: 415-424.

Nakao M, Yanagida T, Okamoto M, Knapp J, et al. (2010a). State-of-the-art Echinococcus and Taenia: Phylogenetic taxonomy of human-pathogenic tapeworms and its application to molecular diagnosis. Infect. Genet. Evol. 10: 444-452.

Nakao M, Li T, Han X, Ma X, et al. (2010b). Genetic polymorphisms of Echinococcus tapeworms in China as determined by mitochondrial and nuclear DNA sequences. Int. J. Parasitol. 40: 379-385.

Nakao M, Lavikainen A, Yanagida T and Ito A (2013). Phylogenetic systematics of the genus Echinococcus (Cestoda: Taeniidae). Int. J. Parasitol. 43: 1017-1029.

Rawson PD and Burton RS (2006). Molecular evolution at the cytochrome oxidase subunit 2 gene among divergent populations of the intertidal copepod, Tigriopus californicus. J. Mol. Evol. 62: 753-764.

Ronquist F and Huelsenbeck JP (2003). MrBayes 3: Bayesian phylogenetic inference under mixed models. Bioinformatics 19: $1572-1574$. 
Scott J, Stefaniak J, Pawlowski Z and McManus DP (1997). Molecular genetic analysis of human cystic hydatid cases from Poland: identification of a new genotypic group (G9). Parasitology 114: 37-43.

Sharma M, Fomda BA, Mazta S, Sehgal R, et al. (2013). Genetic diversity and population genetic structure analysis of Echinococcus granulosus sensu stricto complex based on mitochondrial DNA signature. PLoS One 8: e82904.

Siracusano A, Delunardo F, Teggi A and Ortona E (2011). Host-parasite relationship in cystic echinococcosis: an evolving story. Clin. Dev. Immunol. 2012: Article ID 639362.

Tajima F (1989). Statistical method for testing the neutral mutation hypothesis by DNA polymorphism. Genetics 123: 585-595.

Tamura K, Peterson D, Peterson N, Stecher G, et al. (2011). MEGA5: molecular evolutionary genetics analysis using maximum likelihood, evolutionary distance, and maximum parsimony methods. Mol. Biol. Evol. 28: 2731-2739.

Wang N, Wang J, Hu D, Zhong X, et al. (2013). Genetic variability of Echinococcus granulosus based on the mitochondrial $16 \mathrm{~S}$ ribosomal RNA gene. Mitochondrial DNA 26: 396-401.

Wang J, Wang N, Hu D, Zhong X, et al. (2014b). Genetic diversity of Echinococcus granulosus in Southwest China determined by the mitochondrial NADH dehydrogenase subunit 2 gene. Sci. World J. 2014: Article ID 867839.

Wang N, Xie Y, Liu T, Zhong X, et al. (2014a). The complete mitochondrial genome of G3 genotype of Echinococcus granulosus (Cestoda: Taeniidae). Mitochondrial DNA 22: 1-2.

Yan N, Nie H, Jiang Z, Yang A, et al. (2013). Genetic variability of Echinococcus granulosus from the Tibetan Plateau inferred by mitochondrial DNA sequences. Vet. Parasitol. 196: 179-183.

Yanagida T, Mohammadzadeh T, Kamhawi S, Nakao M, et al. (2012). Genetic polymorphisms of Echinococcus granulosus sensu stricto in the Middle East. Parasitol. Int. 61: 599-603.

Zhang T, Yang D, Zeng Z, Zhao W, et al. (2014). Genetic characterization of human-derived hydatid cysts of Echinococcus granulosus sensu lato in Heilongjiang province and the first report of $\mathrm{G} 7$ genotype of $E$. canadensis in humans in China. PloS One 9: e109059.

Zhong X, Wang N, Hu D, Wang J, et al. (2014). Sequence analysis of cytb gene in Echinococcus granulosus from western China. Korean J. Parasitol. 52: 205-209. 